\title{
La escuela galáctica: prototipo de escuela creativa transformadora
}

Francisco Menchén Bellón *

\section{Resumen}

El sistema educativo actual es anacrónico. La educación tradicional ha tocado fondo: los maestros están desencantados y los alumnos se aburren en clase. Es urgente una educación alternativa que responda a los desafíos que presenta el siglo XXI, en la que se recojan los nuevos avances de la biología, la física cuántica, la neurociencia y las nuevas tecnologías. LA ESCUELA GALÁCTICA, como prototipo de Escuela Creativa Transformadora, nace con el propósito de crear un nuevo ser humano. El espíritu de la Escuela Galáctica se analiza con amplitud en los pilares fundamentales de esta modalidad educativa.

Palabras clave: Educación. Educación alternativa. Escuela Galáctica. Creatividad. Conexión en redes. Campos energéticos. Calidad.

\section{Galactic school: a prototype of creative transformative school}

\begin{abstract}
The current educational system is anachronistic. Traditional education has hit bottom: teachers are disappointed and students get bored in class. An Alternative Education to respond to the challenges of the twenty-first century and to reflect the new developments in biology, quantum physics, neuroscience and new technologies, its urgently needed. GALACTIC SCHOOL, as a prototype of Creative Transformative School, is founded with the purpose of creating a new human being. The spirit of the Galactic School is fully analysed in the basic pillars of this teaching method.

Key words: Education. Alternative Education. Galactic School. Creativity. Networking. Energy fields. Quality.
\end{abstract}

\section{Introducción}

¿Sabes que cada ser humano es un artista que tiene una obra que crear?: su propia vida.

El alumno ha vivido durante mucho tiempo con un desconocimiento total de su propio potencial humano, situación que le ha llevado a vivir una vida limitada,

* Inspector de educación. Explorador de Creatividad (Espanha). E-mail: fcomenchen@yahoo.com 
basada en la competencia, el miedo, el aburrimiento y la escasez, repitiendo lo que decían los libros y los profesores, a base de memorizar. Sin embargo, hoy día sabemos que tiene una capacidad creativa ilimitada, así como un poder inmenso de curiosidad, intuición e imaginación que le permite explorar la realidad. De todas las posibilidades creativas que un alumno pudiera llevar a cabo, existe una que abarca a todas las demás: su propia vida.

Los jóvenes se mueven entre el rechazo a la sociedad y su refugio en su mundo virtual. Millones de estudiantes, a lo largo del mundo, se reúnen sólo para compartir la música, para estar juntos; se niegan a asumir su papel, no porque sean unos desviados sociales sino porque sienten que la sociedad no tiene derecho a pedirles una estabilidad que no confiere ninguna de las grandes instituciones modernas: el parlamento, las autoridades, la empresa, la familia, la escuela, el trabajo y la sociedad atraviesan su más honda y larga de las crisis de identidad.

El ser humano ha sido creado a imagen y semejanza de un ser superior, por ello tiene una capacidad creativa infinita. La mayor creación del ser humano es la Historia de su vida. Se necesitan cambios importantes para avanzar en el siglo XXI, por eso nos atrevemos a proyectar LA ESCUELA GALÁCTICA, como prototipo de Escuela Creativa Transformadora, donde los alumnos vivan no para competir sino para conectarse con los otros, la vida, la naturaleza, el cosmos; donde el corazón no viva perseguido por el miedo, en un ambiente agresivo, sino en un estado de confianza en sí mismo y en los demás que le permita crecer en armonía; donde el aburrimiento y la escasez sean sustituidos por el entusiasmo y la abundancia. Todo ello exige diseñar un nuevo paradigma de modelo educativo, que integre la dimensión física, mental, energética, sutil y espiritual.

Las Facultades de Educación han crecido y se han desarrollado a luz de la doctrina de Newton y Descartes, figurando sus principios como verdades ineludibles de la enseñanza en todos los niveles educativos, durante un siglo, aproximadamente. Este modelo ya no es compatible con los nuevos descubrimientos científicos y tecnológicos.

Si queréis saber qué rumbo tomará la educación y las disciplinas afines como la pedagogía, la psicología, la didáctica y la organización escolar, durante el próximo cuarto de siglo, en la Formación del Profesorado, hemos de proyectar una nueva mirada profunda, llena de energía creativa, que sea capaz de generar un bienestar extraordinario en todas las dimensiones de la vida escolar.

Se necesita una nueva mirada y un nuevo enfoque, porque la educación tradicional es anacrónica, está ya caduca, periclitada, obsoleta; hemos de buscar una educación creativa y además transformadora. Es necesario una transformación radical y 
positiva en la condición humana, una transformación que va más allá de la educación formal. Una transformación que afectará a la sociedad de una forma tan profunda, que ocurrirá lo mismo que sintieron los ciudadanos del Renacimiento, que vino a cambiar el curso de la civilización europea. Es necesario un terremoto cultural, un big bang, un tsunami que trastorne por completo la sociedad.

Lo que resulta deleznable es dar por hecho que la educación oficial es la única modalidad de educación. Esto sería como pensar que los violinistas son los únicos músicos de la orquesta. La educación convencional es la que nos ha llevado a esta tremenda crisis silenciosa, como diría Nussbaum, distinguida en el año 2012 con el Premio Príncipe de Asturias de Ciencias Sociales.

El objetivo que se pretende alcanzar con esta aportación es resaltar que la educación actual está diseñada para formar trabajadores válidos para la era industrial, modelo inadecuado para la era del conocimiento y digital. Mi intención es promover un la Escuela Creativa Transformadora, que mediante la formación del cuerpo, mente y alma desarrolle los talentos del niño, para alcanzar su plenitud.

El modelo de Escuela Creativa Transformadora está inspirado en la filosofía que se proyecta en la RED INTERNACIONAL DE ESCUELAS CREATIVAS (RIEC), que se concreta en el cumplimiento de unos indicadores que se recogen en el INSTRUMENTO PARA VALORAR EL DESARROLLO CREATIVO DE INSTITUCIONES EDUCATIVAS (VADECRIE) de Saturnino de la Torre (2012), promotor y líder de este proyecto. Siguiendo este protocolo hay ya ciertas escuelas que han sido reconocidas, como el Colegio BRÜNING de Lima (Perú) y la Escuela Básica Municipal VISCONDE DE TAUNAY de Blumenau (Brasil), ambas distinguidas en 2013.

En este sentido, surge como prototipo la Escuela Galáctica, ya que cumple los criterios esenciales que requiere dicha Red: transcender, recrear, innovar, valorar $y$ transformar. La intención es conseguir que el alumno alcance una nueva conciencia que le permita evolucionar, a través de la creatividad, el amor y la belleza, imprescindible para el desarrollo pleno del Ser.

\section{La escuela galáctica}

¿Sabes que el sistema educativo actual no desarrolla al ser humano en su plenitud?

Nos encontramos ante un cambio de paradigma, que significa un cambio de mentalidad, lo que supone modificar completamente nuestra forma de pensar, sentir 
y actuar, es decir, nuestro estado del Ser. Tarde o temprano, tendremos que abandonar muchos de nuestros antiguos hábitos y adoptar otros nuevos. Vivimos momentos sociales de grandes cambios y grandes transformaciones; es el mejor momento para que la Escuela Galáctica, la Escuela Creativa Transformadora, florezca y así recuperar pronto la creatividad, el amor y la alegría de vivir.

Aprender ya no es sinónimo de apropiación pasiva de "verdades" inmutables de la escuela tradicional, sino una actividad poiética, es decir productiva, creativa y transformadora, en la que estamos involucrados tanto los maestros e instituciones educativas, como la familia, las empresas, las autoridades y la sociedad en general. Todos ellos están en interacción con un medio ambiente en permanente transformación.

Transformar significa cambiar, en términos de modificar para mejorar, progresar y evolucionar. Este camino de transformación implica cambios estructurales que permita alcanzar la dimensión creativa y espiritual del ser humano, quien fue creado para dar y recibir amor y vivir en armonía. La creatividad individual está constituida por una energía tan poderosa que revierte al creativo y lo regenera. “... liberar la imaginación de los impedimentos o las trabas que pueden poner a veces las palabras, las categorías o las etiquetas (Bachrach, 2013a, 35-6).

El proceso de transformación creativa es el proceso de superar el miedo. Alonso Puig (2012), médico, especialista en liderazgo y creatividad, explicaba en una conferencia: "Nos da mucho miedo cambiar porque sabemos lo que perdemos, lo que no sabemos es lo que podemos ganar. Y, cuando se alza la barrera del miedo, tendría también que alzarse ese caudal de energías dormidas, de potencial oculto". Hay que reconocer que el miedo es la creencia que no podemos crear, y que esa creencia es una ilusión.

Hoy más que nunca se hace necesario la presencia de la Escuela Galáctica, con maestros galácticos, provistos de una nueva mirada, evolucionada, que conecte con la naturaleza, con la energía del cosmos, con el alma, y la espiritualidad, capaz de inspirar a sus alumnos un nuevo despertar a la esencia de la vida, descubriendo el valor del poder interior. Hemos perdido el contacto con la naturaleza, lo que significa perder el contacto con nosotros mismos, porque somos parte de ella.

\section{¿Sabes que el aprendizaje auténtico}

lleva consigo cambios significativos en la actitud?

La topografía de la Escuela Galáctica responde al modelo 7 / 24, donde toda la sociedad debe estar implicada en la educación de los alumnos, las veinticuatro horas del día, los siete días de la semana. Será necesario toda la tribu, pero especialmente 
la competencia de los maestros que se entusiasmen con su trabajo, pues ellos serán el baluarte más significativo para mirar el futuro.

No habrá educación creativa si no pensamos que el alumno es creativo y necesita liberar su creatividad. La creatividad no es patrimonio de unos pocos sino una característica central del ser humano que debe estar presente en todos nuestros actos y pensamientos para dar continuidad a la vida.

Para que la Escuela Galáctica sea una realidad es necesario contar con una mutación espectacular, en las que estructuras enteras se pierdan y sean sustituidas por otras. O como diría Richard Dawkins (2002) o Ken Wilber (2011) que en las nuevas generaciones se activen ciertos memes y se desactiven vías enteras de desarrollo, es decir una metamorfosis; se definen los memes como "unidades de herencia cultural", comparable a los genes de la biología; son ideas que se contagian de manera vertiginosa de unos seres humanos a otros. "Los memes... se transmiten de cerebro en cerebro a través de un proceso que, en un sentido amplio, puede llamarse imitación... Los memes deberían considerarse estructuras vivas" (citado por Sheldrake, 2006a, 374).

Nuestra sociedad está viviendo una acelerada mutación, pero los docentes no están preparados para alcanzar este nivel de evolución. Lo único que nos impide dar este salto cualitativo es nuestro propio miedo, como ya hemos señalado, por lo que hay que aprender a dominar el miedo y hacernos creativos y confiar en nuestras posibilidades, utilizando el cerebro cuántico, responsables de las ideas novedosas y originales; tendrán que surfear entre las dificultades, emociones, miedos...y aprender a fluir entre ellos.

La Escuela Galáctica se sitúa en contra de la educación transmisiva y conservadora, manteniendo una fuerte conexión entre la acción educativa y la acción social, a través del diálogo, considerado como un encuentro pedagógico esencial. La creatividad no puede ir en línea recta, está en un continuo vaivén que va del inconsciente al consciente, mientras el sujeto percibe como uno alimenta al otro.

¿Sabes que el miedo paraliza a los docentes implicarse en el futuro, $y$ prefieren continuar en la zona de confort?

El miedo nos hace creer que no somos capaces de crear y ello nos obliga a continuar en nuestra zona de confort. El miedo inhibe la autoestima y la confianza, paraliza la creatividad, e impide emprender e implicarse en el futuro. Los especialistas en psicoespiritualidad dicen que el único antídoto para el miedo es el amor, no el que recibimos, sino el que damos. Los grandes científicos como Newton, Einstein, 
y Schröedinger, entre otros, eran sumamente místicos y aprendieron a dominar sus miedos. Hay que cambiar el enfoque educativo: dominar el miedo y maximizar la creatividad propia y la de los demás; es una referencia a la autopoiesis de los biólogos chilenos Varela y Maturana.

\section{Pilares de la escuela galáctica}

¿Sabes que puedes imaginarte un mundo en el que los seres humanos tienen una relación natural e instintiva mediante la colaboración y el amor, no mediante la competitividad y la envidia?

El sistema educativo debe ofrecer otras alternativas, distintas al modelo establecido, para que los alumnos de todas las edades puedan tener la opción de ser creativos, transcendentes y espirituales. Si observamos a cualquier niño, antes de los seis años, podemos afirmar que son creativos desde el nacimiento. Pero luego la escuela y la sociedad deja de estimular las conexiones neuronales propias de la creatividad, y en su lugar empiezan a usar los circuitos de la lógica, la razón y el análisis, que se convierten en el patrón de pensamiento dominante.

Nos encontramos ante un cambio de paradigma, que significa un cambio de mentalidad, lo que supone modificar completamente nuestra forma de pensar. Tarde o temprano, tendremos que abandonar muchos de nuestros antiguos hábitos y adoptar otros nuevos. Vivimos momentos sociales de grandes cambios y grandes transformaciones; es el mejor momento para que la Escuela Galáctica florezca.

Necesitamos una escuela con pedigrí, que despierte en los alumnos el entusiasmo por ir a ella y en los docentes por trabajar con ilusión. La sociedad en general y las autoridades se deben sentir orgullosas de sus instituciones educativas. En el futuro, la escuela seguirá siendo necesaria, a pesar de que los tecnólogos dicen que la computadora va a hacer volar la escuela.

La Escuela Galáctica reconoce los nuevos descubrimientos del Sistema Creativo del ser humano: el cerebro no solo es un dispositivo de almacenamiento de memoria, sino que empieza a considerarse como un sofisticado sistema de sintonización e integración de señales; el corazón no solo es el centro de las emociones, sino el núcleo más poderoso de energía electromagnética, y la conciencia no solo es una, sino también conecta con la conciencia universal. Los pilares que sostienen la Escuela Galáctica confluyen en la presencia de las $5 \mathbf{C} \boldsymbol{s}$ : 
- C reatividad transformadora

- C onciencia integradora

- C onexión en red

- C ampos energéticos

- C alidad suprema

\section{A) creatividad transformadora}

La relación auténtica entre el maestro y el discípulo

se produce cuando el docente vierte su luz en el corazón del discípulo, con el objeto de que tome conciencia

de todo lo que está oculto en su interior

$y$ vaya descubriendo su potencial, el tesoro más preciado.

Osho (2010)

En la Escuela Galáctica, la creatividad cobra vida y recupera el poder de imaginación que es anterior a la lógica; no puede ir en línea recta, está en continuo vaivén que va del inconsciente al consciente, mientras el sujeto percibe como uno alimenta al otro. En el ser humano, la creatividad transformadora es ilimitada, posee una flexibilidad infinita y no está contenida en una red rígida de energía e información, obviamente sigue las leyes del universo y está de acuerdo con la propia naturaleza de cada ser.

La creatividad transformadora se produce mediante el cambio de mentalidad y la evolución de la conciencia; supone recrear o reinventarse de forma permanente pues, el individuo no es una realidad acabada, sino un ser en tránsito. Implica cambios estructurales que permitan alcanzar la dimensión espiritual del ser humano, quien fue creado para dar y recibir amor y vivir en armonía.

La creatividad transformadora de maestros y alumnos convertirán la Escuela Galáctica en un gigantesco laboratorio de nuevas posibilidades cognitivas, donde concurran los procesos convergentes y divergentes. Hay que crear nuevos saberes para un mundo que reconoce la emergencia de lo nuevo. A través de la creatividad transformadora el alumno aprenderá a construir y recrear el conocimiento y saber aplicarlo a su entorno, transformando su realidad socio-cultural, de tal forma que la práctica esté ligada a referentes emancipadores. Estos conocimientos generados desde un nivel de conciencia responsable son significativos y no se olvidan.

Las nuevas generaciones de alumnos vienen con un sistema físico, mental y energético mejor adaptado y rinden más, y son más creativas que las promociones 
anteriores. Dada la plasticidad del cerebro, con entrenamiento adecuado, una persona puede cambiar su programación, mejorar el procesador, aumentar el número de conexiones neuronales y optimizar el funcionamiento cerebral, lo que se traduce en una mayor creatividad e innovación. Esto brinda un número mayor de posibilidades, al gestionar el cerebro más cantidad de información simultáneamente

\section{B) Conciencia integradora}

"El hombre no puede entender dónde está, porque ha creado dos mundos a partir de uno"

Ken Wilber $(2011,47)$

La Escuela Galáctica pretende despertar la conciencia para reconstruir la dignidad del ser humano, despertar el amor y la belleza del universo. No vemos lo que se encuentra en el exterior si no lo hemos encontrado primero interiormente. Todo lo que somos capaces de percibir, no es más que un reflejo de nuestro mundo interior. No es fácil el acceso a este nivel de conciencia, porque nuestra mente, durante miles de años, ha sido educada por leyes eternas y no ha contemplado el concepto de evolución y espiritualidad.

La conciencia integradora se ocupa, entre otras cosas, de unificar y armonizar los opuestos, tanto positivos como negativos, descubriendo un fundamento que trascienda y abarque a ambos. Ken Wilber recomienda liberar los pares de opuestos, buscar la reconciliación, entender la unidad de estas polaridades y tener conciencia de ellos, porque la realidad no es dual, no tiene fronteras, sino una unión de opuestos. El dualismo es el punto de vista de la civilización occidental. Biológicamente, no hay ningún fundamento para esta disociación o escisión radical.

Citado filósofo norteamericano nos recuerda que, el mundo de los opuestos es un mundo de conflictos. Es posible que los opuestos sean diferentes como "comprar y "vender", pero la esencia es que son acontecimientos completamente inseparables, son simplemente dos aspectos de un solo hecho, es decir, la transacción como tal. De la misma manera, todos los opuestos comparten una identidad implícita y recíprocamente dependiente, porque ninguno de ellos podrá existir sin el otro $(2011,36)$.

"La realidad está más allá de nombres y formas, de palabras e ideas, de divisiones $y$ límites" (Ken Wilber, 2011, 69). La realidad que creamos con nuestra observación, es conciencia, crea un espacio y un tiempo que también son conciencia; resulta ser una proyección de la conciencia que trasciende. El espacio y el tiempo de nuestra existen- 
cia están ligados a la conciencia. De modo que el entorno, mediante nuestra observación, se vuelve consciente, lleno de vida y de posibilidades. Somos seres universales. La evolución del universo es la evolución de la conciencia.

La conciencia humana, a nivel cuántico, es infinitamente flexible, y tiene la habilidad de poder cambiar conscientemente el contenido de información, que da origen a nuestro cuerpo físico, e influir en el contenido de energía y de información de nuestro cuerpo ampliado, en consecuencia, hacer que se manifieste lo que deseamos. Dicho de otra forma: materializar un deseo. Somos conciencia materializada, nuestros componentes son los mismos ladrillos que conforman el sol, los planetas, el mar y la tierra. En este sentido somos como el caracol, que vaya a donde vaya, siempre está en su casa.

Cada individuo ha de sentir que tiene una misión en la vida y que tiene algo especial que ofrecer, y debe pensar: por qué estoy en la Tierra; este mensaje viene directamente del alma y entra en la conciencia física. No se puede utilizar el cuerpo solo para el disfrute y el placer sensual, sin dar ninguna importancia al alma. El sistema educativo debe contemplar la posibilidad de aprender a pensar, pero también debe inculcar a que el alumno aprenda a sentir su conciencia interna, así como los sentimientos y emociones que provoca el mundo exterior.

\section{C) Campos energéticos}

"Los campos son el medio de "acción a distancia"
y a través de ellos los objetos se afectan entre sí
aun sin estar en contacto material"
Rupert Scheldrake $(2006,158)$

Citado científico británico, postula que cada especie tiene un «campo» de memoria propio. Este concepto está incorporado en este nuevo paradigma, con la intención de aplicarlo a la educación, siendo uno de los signos de identidad de la Escuela Galáctica. La escuela no puede estar aislada, está afectada por todo tipo de campos, ya provengan de la naturaleza, del mundo físico, biológico, psíquico o cultural; todos ellos campos energéticos. Nuestra vida transcurre constantemente en estos campos.

Einstein al elaborar la teoría de la relatividad restringida (1905) empleó este término, indicando que existen de forma inmaterial en la naturaleza. No son objetos materiales, sino zonas de influencias. Por ejemplo, el campo que se crea alrededor de un imán es invisible, pero su existencia puede comprobarse esparciendo unas cuantas limaduras de hierro alrededor, son cuantos de energía vibracional. 
El cuerpo humano es un campo de energía e información estructurada de ondas estacionarias. Este campo está constituido por las formas y actitudes de todas las personas que vivieron en el pasado de dicha especie, y su influencia moldea a todos los individuos futuros: cada especie animal, vegetal o mineral posee una memoria colectiva a la que contribuyen todos los miembros de la especie y a la cual conforman.

Un campo energético de ondas está organizado proporcionalmente en niveles de jerarquía. El corazón irradia un campo que no solo influye en todos los demás órganos sino también en el entorno. El corazón está constantemente emitiendo ondas: sonido, calor, presión, señales electromagnéticas, luz y cada célula del cuerpo recibe en un determinado momento esta influencia vital.

Las formas de todos los miembros pasados de nuestra especie «resuenan» en nosotros, como ondas en un estanque, organizando la vía de nuestro crecimiento. A la vez, nosotros incorporamos nuestra forma a la memoria colectiva de la especie, engrosándola e incrementando así su influencia. Y al igual que las formas, también «resuenan» todo tipo de instintos y actitudes. A esto se le llama resonancia mórfica, un proceso mediante el cual el pasado se hace presente. Es el modo por el cual la información se transmite instantáneamente entre miembros de una misma especie, más allá del espacio-tiempo. Nuestras experiencias y nuestras habilidades adquiridas no se pierden. La información vuelve al campo donde es reutilizado por otros miembros de la especie.

La resonancia mórfica tiene fuerte implicaciones en la educación y abre caminos insospechables para un aprendizaje totalmente novedoso. Los niños de las sucesivas generaciones aprenden con más facilidad las habilidades y conocimientos de sus antecesores. Nuestras ideas y actitudes influyen a distancia sobre otras personas, aún sin darnos cuenta. Nuestra mirada, sentimientos y experiencias modifican los campos mórficos y de energía, a su vez estos campos nos influyen y modelan constantemente.

\section{D) Conexión en red}

"La red es un patrón común a todo lo vivo.

Allí donde hay vida, hay redes"

Fritjof Capra $(2003,32)$

La Escuela Galáctica debe contar con un centro de conexión en red, de tal forma que le permita estar unida al cosmos, por un lado, y al ser humano por otro. El pensamiento es una onda que transporta energía e información. Esta onda de información 
tiene el poder de crear, pero también de eliminar los programas nocivos y regenerar los tejidos.

El cerebro gracias a su plasticidad neuronal es capaz de dinamizar la energía y crear nuevas redes, con la consiguiente apertura de creatividad. La conexión en red permite la creación de nuevas semillas, de cara al futuro. El cerebro es una superbiocomputadora. Nuestro cuerpo no es independiente del cuerpo del universo, porque más allá de nuestros átomos y partículas elementales, las fronteras no están bien definidas. Nuestro cuerpo no está limitado por la piel. Estamos interconectados con todo. Este es tan solo un nivel que delimita un medio interno y otro externo, esto le permite al cuerpo funcionar y desarrollarse como organismo.

Tenemos que observar las estructuras y funciones de nuestro cuerpo, como resultado de la interacción de una enorme población de células inteligentes, y detectaremos que todas ellas conectan en red y armonizan con el resto y trabajan en conjunto, renunciando a su individualidad cuando las condiciones lo requieren, pero sin perder sus habilidades y su información. La conexión en red constituye un centro magnético muy potente que amplifica las probabilidades para innovar.

En una célula se producen cerca de 100.000 reacciones químicas por segundo, incluso la información acerca del lugar y el momento exacto en que estas reacciones químicas se han de producir. Un verdadero láser endógeno. Erwin Schröedinger, que recibió en 1933 el premio Nobel de física y es considerado como uno de los creadores de la física cuántica, declaró que un ser vivo sólo puede mantenerse en un nivel alto de orden porque recibe continuamente mensajes de su entorno. Es la luz del sol la que crea en definitiva este orden.

E) Calidad suprema

Ban Ki-moon, secretario general de la ONU, en la presentación de la campaña

la educación ante todo,

destacó como una de las prioridades a realizar

mejorar la calidad del aprendizaje

(26 de Septiembre 2012)

La Escuela Galáctica debe dejar huellas, aunque muchas veces sean invisibles. Somos seres llenos de pasión, pero la mayoría de las veces no se manifiesta. Cuando el trabajo se hace con entusiasmo y devoción, los resultados trascienden, se valoran y alcanzan la calidad suprema, la expresión óptima, la excelencia. La educación que se 
ha de ofrecer a los niños y a los jóvenes debe estar dirigida a pensar en las necesidades de la sociedad o en los anhelos propios, pero no, exclusivamente, en ser famoso y ganar dinero.

Si prestamos atención a algo le transferimos energía, ya que el pensamiento es una onda de energía e información y entonces el objeto de atención se manifestará con más fuerza en nuestra vida, consiguiendo la máxima calidad. Si dejamos de prestar atención, se marchitará, se desintegrará y desaparecerá.

Los pensamientos, cuando conectan con el corazón, si se le pone pasión, pueden alcanzar la calidad suprema. El alumno debe asombrarse y emocionarse, de tal forma que se ilumine su alma y se le ericen los pelos, para que aparezca el lado poético de la vida. La dimensión sutil está muy conectada con el plasma emocional.

El alumno que asiste a la Escuela Galáctica encontrará algo mágico que le sorprenderá. La tarea principal que tiene es descubrir, explorar e indagar, ignorando los caminos convencionales; esto es un juego muy divertido, porque el maestro no le da pistas. Cuando descubre algo bello que no esperaba, algo que estaba allí y que pasó por alto, le parece que hay magia. No tenemos que olvidar que el doctor Fleming descubrió la penicilina por accidente.

En las Escuelas Creativas los alumnos son como paleontólogos que cavan y escarban pacientemente, hasta que se topan con algo desconocido que es valioso. Los maestros galácticos reconocen lo mejor de sus alumnos que crecen por dentro y por fuera, buscando en todas las tareas la máxima calidad.

Sir Ken Robinson (2009), experto mundial en el desarrollo del potencial humano, a su interesante libro El Elemento, le ha puesto el subtítulo Descubrir tu pasión lo cambia todo. Este famoso autor nos recuerda, repetidas veces, que cuando disfrutamos haciendo aquello que más nos apasiona, se alcanza la máxima calidad, a la vez que se desarrolla la creatividad. El problema es que no somos conscientes de nuestros talentos e inclinaciones naturales.

Un pensamiento positivo puede ser muy poderoso. La calidad del pensamiento es la imagen de la posibilidad cuántica. En el reino de las posibilidades todo existe, solo que de manera no manifestada. Todas las posibilidades existen superpuestas y con el pensamiento escogemos una de tantas. Podemos identificarla y aislarla. Pero para poder insuflarle calidad de vida hace falta el sentimiento. Para que esa posibilidad cuántica se realice en la realidad cotidiana hay que agregarle el sentimiento: amor o miedo, los dos funcionan, aunque el miedo es menos poderoso y genera otros efectos adversos. No hace falta saberlo, es suficiente con sentirlo.

La escuela necesita un cambio orgánico y discontinuo para que produzca un 
impacto y sea una auténtica evolución, no un fenómeno de laboratorio, ni una tarea de despacho. Todo el progreso y toda la riqueza humana vienen de la mano de la creatividad. Las personas no creativas se convertirán en parásitos. La creatividad crea a(C) tividad.

\title{
Reflexiones finales
}

\author{
"Creemos en una escuela que despierte \\ los sueños de nuestra juventud, \\ que cultive la solidaridad, la esperanza \\ $y$ el deseo de aprender, enseñar y transformar el mundo" \\ (Palabras colgadas en la pared de las fincas agrarias \\ ocupadas por el Movimiento de los trabajadores sin Tierra de Brasil) \\ ¿Sabes que somos seres emocionales que aprendimos a pensar \\ y no máquinas pensantes que sentimos?
}

Los maestros y profesores de los distintos niveles educativos no pueden soportar otro siglo XX, están desmoralizados, han perdido la ilusión por la enseñanza, pues el sistema ha destruido ese poder inmanente que le daba su experiencia, así como el sentido vocacional que le permitía modelar una mente sana, vigorosa, transparente y creativa. Todos ellos necesitan resetear su cerebro y empezar a conocer y enamorarse de los atractivos de la Escuela Galáctica.

Es urgente la necesidad de LA ESCUELA GALÁCTICA que se preocupe de crear un hombre nuevo con una conciencia nueva, ello supone enfocar la propuesta guiados por la intuición, analizar las creencias limitantes, activar los dos hemisferios cerebrales, atreverse a entrar en espacios invisibles, desconocidos e imposibles y confiar en nosotros mismos.

Para que se encienda la luz de una mente nueva deben cuidarse con detalle los elementos que componen el Sistema Creativo del ser humano: c erebro, c orazón, c onciencia, $\mathbf{c}$ reencias y c uerpo, (las $5 \mathrm{Cs}$ ): todo un arte. Ramón y Cajal, premio Nobel de Medicina en 1906, opina: "Todo ser humano puede ser, si se lo propone, escultor de su propio cerebro"

La Escuela Galáctica cumple los requisitos de la Escuela Creativa Transformadora. La Escuela Galáctica transciende porque busca ir más allá y deja huella; recrea e innova porque cada sujeto aspira a construir su propia vida; valora, porque los alum- 
nos aprenden a dar sentido a la vida y a disfrutar de la belleza de nuestro planeta $y$ transforma porque todos los actores del hecho educativo son conscientes de la importancia que tiene evolucionar.

La Escuela Galáctica deberá alinear el cuerpo, le mente y el espíritu, porque todo está interconectado: deberá aceptar el poder de ser el creador de sus sueños y de sus circunstancias; deberá aprender a manejar sus emociones sin juzgarlas; deberá cambiar la imagen limitante que tiene de sí mismo y crear desde dentro la vida que desea tener; deberá ser el director y protagonista de su propio destino. Todo ello, le conducirá a descubrir una conciencia sutil, culta y virtuosa que cambiará su vida para siempre.

Los pilares de la Escuela Galáctica nos muestran que es posible alcanzar nuevos estados de conciencia, es decir, evolucionar, permitiendo crear una nueva realidad, valorando la vida, el aprendizaje, las relaciones y alcanzando la calidad óptima, debido a las nuevas conexiones neuronales que se establecen.

La Escuela Galáctica proyectará una nueva visión del mundo y funcionará como un todo, impregnando al alumno de una conciencia planetaria, donde su corazón sincronizará con el universo y experimentará el amor universal. El sentido de nuestra existencia forma parte de un engranaje colosal que se desarrollará en perfecto orden y armonía, pues el mundo existe dentro de nosotros, cuando funcionamos como un todo, pero no somos conscientes.

La Escuela Galáctica, como prototipo de Escuela Creativa Transformadora, viene a trascender, recrear, innovar y transformar. Para ello, hay que activar el cerebro y sanear las carencias del modelo tradicional, así como dar esplendor a los talentos naturales de los alumnos, intentando que cada uno consiga la mejor versión de sí mismo, tratando de optimizar los distintos niveles de aprendizaje.

\section{Referências}

ALONSO PUIG, M. Reinventarse. Tu segunda oportunidad. Barcelona: Plataforma, 2010.

. Ahora yo. ¿Y si creas tu propio futuro en lugar de encontrarlo? Barcelona: Plataforma, 2011.

BACHRACH, E. Ágil Mente. Aprende cómo funciona tu cerebro para potenciar tu creatividad y vivir mejor. Barcelona: Conecta, 2013. 
CAPRA, F. Las conexiones ocultas. Implicaciones sociales, medioambientales, económicas y biológicas de una nueva visión del mundo. Barcelona: Anagrama, 2003.

CHOPRA, D. Energía sin límites. Barcelona: Byblos, 2006.

. Sincrodestino. México: Alamah, 2007.

. Reinventa tu cuerpo, resucita tu alma. Cómo crear un nuevo yo. Madrid: Aguilar, 2010.

. Soluciones espirituales. Respuestas a los mayores desafíos de la vida. Barcelona: Ediciones B, 2012.

CHURCH, D. La medicina energética y la nueva biología de la intención. Barcelona: Obelisco, 2008.

DAWKINS, R. El gen egoísta. Las bases biológicas de nuestra conducta. Barcelona: Salvat, 2002.

DISPENZA, J. Desarrolla tu cerebro: la ciencia de cambiar tu mente. Madrid: La Esfera de los libros, 2008.

. Deja de ser tú. La mente crea la realidad. Barcelona: Urano, 2012.

GOSWAMI, A. El médico cuántico. Guía de la física cuántica para la salud y la sanación. Barcelona: Obelisco, 2009.

LIPTON, B. H. La biología de la creencia. Madrid: La Esfera de los Libros, 2007. . La biología de la transformación. Madrid: La Esfera de los Libros, 2010.

MENCHÉN, F. Descubrir la creatividad. Desaprender para volver a aprender. Madrid: Pirámide, 2002. . La creatividad en el aula. Perspectiva teórico-práctica. Conocimiento: Santiago de Chile, 2008. 
. La Creatividad y las Nuevas Tecnologías en las Organizaciones modernas. Madrid: Díaz de Santos, 2009.

. La creatividad transforma la realidad. En Revista Educación y Futuro. Centro de Enseñanza Superior en Humanidades y Ciencias de la Educación Don Bosco. Madrid. No 21. Octubre, 2009. p. 89 - 110.

. La riqueza del tiempo libre. Cómo activar tu creatividad en el tiempo de ocio. México: Trillas, 2011.

- A Escola galáctica. A escola criativa transformadora: Uma nova consciencia. En Torre, S.; Pujol, M.A.; Souza, V. Inovando na sala de aula. Blumenau: Nova Letra. 2013. p. 32-51.

Despierta tu creatividad: Atrévete. En "Escuelas y aulas creativas. Reflexiones y Experiencias". En Pujol, María Antonia, De la Torre, Saturnino, Lorenzo, Nuria (Coord.). VI Fórum Internacional. INCREA. Universitat Barcelona. Institut de Ciéncies de l’Educació, 2014. p. 44-85.

. La necesidad de Escuelas Creativas. La Escuela Galáctica. Una nueva conciencia. Madrid: Díaz de Santos, 2015.

MORA, F. Neuroeducación. Solo se puede aprender aquello que se ama. Madrid: Alianza Editorial, 2013.

NARANJO, C. Cambiar la educación para cambiar el mundo. Vitoria: La llave, 2009. La mente patriarcal. Barcelona: RBA, 2010.

NUSSBAUM, M.C. Sin fines de lucro. Por qué la democracia necesita de las humanidades. Buenos Aires: Katz, 2011.

OSHO Cambio. Cómo convertir una crisis en una oportunidad. Barcelona: Grijalbo, 2010. 
. No tienes nada que perder. En el camino hacia la verdad, la conciencia y la felicidad. Barcelona: Debolsillo, 2010.

ROBINSON, K. y ARONICA, L. El Elemento. Descubrir tu pasión lo cambia todo. Barcelona: Grijalbo, 2009.

. Busca tu elemento. Aprende a ser creativo individual y colectivamente. Barcelona: Empresa Activa, 2012.

SALAS, D. Moneda cósmica. La suprema riqueza. Alicante: Agua Clara, 2011.

SHELDRAKE, R. Caos, creatividad y conciencia cósmica. Castellón: Ellago, 2005.

. La presencia del pasado. Resonancia mórfica y hábitos de la naturaleza. Barcelona: Kairós, 2006.

TORRE, S. Dialogando con la creatividad. De la identificación a la creatividad paradójica. Barcelona: Octaedro, 2003.

- y MORAES, M.C. Sentipensar. Fundamentos y estrategias para reencantar la educación. Málaga: Aljibe, 2006.

. y VIOLANT, V. Comprender y evaluar la creatividad. Un recurso para mejorar la calidad de la enseñanza. Vol. 1. Málaga: Aljibe, 02006.

TORRE, S. Instrumento para valorar el desarrollo creativo de instituciones educativas (VADECRIE). El Ejido (Almería): Círculo Rojo, 2012.

WILBER, K. La conciencia sin fronteras. Aproximaciones de Oriente y Occidente al crecimiento personal. Barcelona: Kairós, 2011.

STREAMING. ÁgilMente: aprende cómo funciona tu cerebro para potenciar tu creatividad y vivir mejor. Disponível em: www.youtube.com/watch?v=OufczQfFq74 Acesso em: 23/07/13. 
WEBISLAM. Conferencia del Manuel Arrieta. Disponível em: http://www.webislam. com/videos/58156-manuel_arrieta_medico_y_chaman.html Acesso em: 26/07/13.

CREANDOTUVIDA BLOG. Disponível em: www.creandotuvidablog.com Acesso em: 25/08/13.

SOCIEDAD TEOSÓFICA ESPANÕLA. Disponível em: www.sociedadteosofica.es/ Artículos Acesso em: 29/08/13.

Recebido em: 29 março 2016.

Aceito em: 06 abril 2016. 